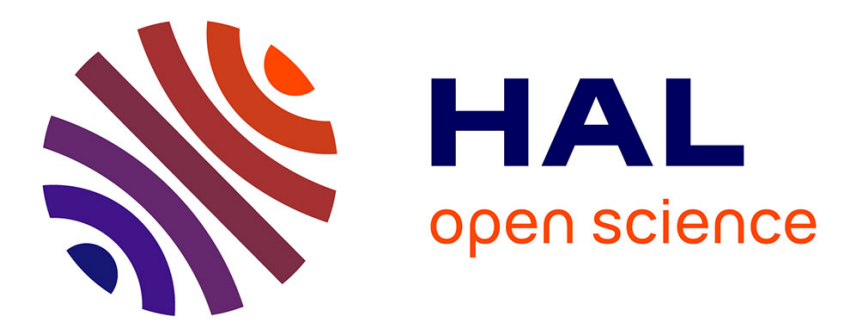

\title{
Gas transport in fibrous media: Application to in-plane permeability measurement using transient flow
}

Yi Hou, Sébastien Comas-Cardona, Christophe Binetruy, Sylvain Drapier

\section{To cite this version:}

Yi Hou, Sébastien Comas-Cardona, Christophe Binetruy, Sylvain Drapier. Gas transport in fibrous media: Application to in-plane permeability measurement using transient flow. Journal of Composite Materials, 2013, 47 (18), pp.2237-2247. 10.1177/0021998312455676 . emse-00959603

\section{HAL Id: emse-00959603 \\ https://hal-emse.ccsd.cnrs.fr/emse-00959603}

Submitted on 15 Jul 2021

HAL is a multi-disciplinary open access archive for the deposit and dissemination of scientific research documents, whether they are published or not. The documents may come from teaching and research institutions in France or abroad, or from public or private research centers.
L'archive ouverte pluridisciplinaire HAL, est destinée au dépôt et à la diffusion de documents scientifiques de niveau recherche, publiés ou non, émanant des établissements d'enseignement et de recherche français ou étrangers, des laboratoires publics ou privés.

\section{(ㄷ)(1)}

Distributed under a Creative Commons Attribution| 4.0 International License 


\title{
Gas transport in fibrous media: Application to in-plane permeability measurement using transient flow
}

\author{
Y Hou ${ }^{1,2}$, S Comas-Cardonal,3, C Binetruy ${ }^{1,3}$ and S Drapier ${ }^{2}$
}

\begin{abstract}
This article introduces a methodology to measure in-plane permeability of fibrous media using a transient one-dimensional air flow with absolute pressures ranging from $10^{3}$ to $10^{5} \mathrm{~Pa}$. The method, based on the measurement of gas pressure at the boundaries throughout the transient flow, is convenient, clean and fast, avoids usage of a gas flow meter and offers a way to study the gas transport within fibrous media. The transport of a compressible fluid is described by several models to comply with different flow regimes which can occur during the experimental measurements. $A$ thermal analysis is given to verify the validity of isothermal conditions during the tests. The permeability, only depending on the fibrous structure, is determined by inverse method, fitting the simulation results to the experimental data obtained using raising or dropping pressure methods. The deviation from Darcy's law caused by gas sliding effect is analysed and a relative parameter of fabric material shows a dependence in permeability, with a similar trend as the Klinkenberg sliding parameter in soils and rocks.
\end{abstract}

\section{Keywords}

Fibrous media, sliding flow, permeability, gas flow

\section{Introduction}

Permeability of a fibrous reinforcement used in the structural composite community is an important physical parameter when resin filling simulation of liquid composite moulding has to be performed. Most of the permeability measurement techniques rely on liquid injection experiments ${ }^{1}$ and still show high discrepancies.

In order to reduce this experimental discrepancies, one option is to simplify as much as possible the experimental bench to avoid errors from accumulating. With such considerations in mind, set-ups based on compression of saturated fabrics have been built. $^{2-4}$ To further bound the permeability, instead of switching techniques (injection vs. compression), the nature of fluid can also be switched (fluid vs. gas). ${ }^{5-11}$ Experimentally, compared with liquid measurement, the use of gas has an advantage of short experimental time due to low gas viscosity, and hence, gas permeability measurement has been extensively applied to materials with small permeabilities, such as rocks, soils, membranes and ceramics. ${ }^{12-15}$
Following a review of theoretical and experimental aspects of gas flow in porous media ('Fundamentals on gas flow through porous media' and 'Review on permeability measurements using gas' sections), this article proposes a methodology to measure fabric inplane permeability using a transient one-dimensional (1D) gas flow using vacuum ('Permeability measurement of fibrous material using vacuum' section). A thorough study of the transport of a compressible fluid is performed. Several models based of Darcy's law and sliding regimes are investigated for

\footnotetext{
'Polymers and Composites Technology and Mechanical Engineering Department, Ecole des Mines de Douai, France

${ }^{2}$ Mechanics and Materials Processing Department, LCG UMR CNRS, Ecole Nationale Superieure des Mines, France

${ }^{3}$ Research Institute in Civil Engineering and Mechanics, UMR CNRS 6I83,

Ecole Centrale de Nantes, LUNAM, France
}

\section{Corresponding author:}

$S$ Comas-Cardona, Research Institute in Civil Engineering and Mechanics, UMR CNRS 6183, Ecole Centrale de Nantes, I rue de la Noe, 4432I, Nantes, France.

Email: sebastien.comas@ec-nantes.fr 
fibrous media. A thermal analysis is given to verify the validity of isothermal conditions during the tests. Permeability is extracted by solving the governing equations with an inverse method. Results are given for various types of fibrous materials and compared with liquid injection and saturated compression permeability measurement techniques.

\section{Fundamentals on gas flow through porous media}

Gas transport through porous media is based on four independent mechanisms ${ }^{16}$ :

(a) Viscous flow, in which the characteristic length of pores within the medium is much larger than the mean free path (i.e. the average distance between molecule collisions) so that molecule-molecule collisions dominate molecule-wall collisions;

(b) Free-molecular or Knudsen flow, in which the gas density or the pore size is so low that the number of collisions between molecules can be neglected compared with the number of collisions of molecules with the walls of the porous medium;

(c) Continuum diffusion, in which the different species of a mixture move relative to each other under the influence of concentration gradients, temperature gradients or external forces; and

(d) Surface flow or diffusion, in which molecules move along a solid surface in an adsorbed layer.

For gas permeability measurement of fibre reinforcement, viscous, Knudsen and sliding flow (an interaction regime between viscous and Knudsen flows) dominates, thus diffusions can be neglected. The gas transport regimes are determined by the Knudsen number $K_{n},{ }^{17}$

$$
K_{n}=\frac{\lambda}{l}
$$

where $\lambda$ is the mean free path, which depends on pressure, temperature and gas molecular weight and $l$ the characteristic length of pores within a medium. For gas molecules at standard temperature and pressure, i.e. $25^{\circ} \mathrm{C}$ and $1 \mathrm{~atm}, \lambda$ is approximately $8 \times 10^{-8} \mathrm{~m}$.

\section{Knudsen flow}

When $K_{n}>1$, the molecules do not frequently collide with each other within the pores. The original studies of Knudsen flow were limited to small holes in very thin plates, and molecules were assumed to move entirely independent of each other during their passage through the holes. A Knudsen flow parameter $K_{m}(\mathrm{~m})$, only related to geometry of the hole and the gas-surface scattering law, can be defined, ${ }^{16}$

$$
J_{k}=-K_{m} \bar{v} \nabla \tilde{n}
$$

where $\bar{v}$ is the mean velocity, $\tilde{n}$ the molecular density $\left(\mathrm{mol} / \mathrm{m}^{3}\right)$ and $J_{k}$ the Knudsen flux (mol/(s. $\left.\mathrm{m}^{2}\right)$ ).

\section{Viscous flow}

When $K_{n} \ll 1$, the size of pores within a medium is large enough for molecules to collide frequently. In this case, the flow is continuous, viscous and can be driven by pressure gradients within the pores. A Newtonian viscous flow can be described by the Navier-Stokes equation. Random paths of molecules after collisions with walls induce a no-slip (zero velocity) boundary condition. For a laminar flow with a Reynold's number $R e<0.1$, the inertial term in Navier-Stokes equation can be neglected, and hence, the profile of velocity depends only on the local microstructure. The viscous flux $J_{\text {visc }}\left(\mathrm{mol} /\left(\mathrm{s} \cdot \mathrm{m}^{2}\right)\right)$ is given by, ${ }^{17}$

$$
J_{v i s c}=-\left(\tilde{n} K_{v} / \mu\right) \frac{\Delta P}{\delta}=-\left(\tilde{n} K_{v} / \mu\right) \nabla P
$$

where $\Delta P$ is the pressure difference across the distance $\delta, K_{v}$ the viscous permeability $\left(\mathrm{m}^{2}\right)$ and $\mu$ the fluid viscosity.

In a porous medium where the region of interest is much larger than the representative volume element of the porous medium, the flow can be represented with Darcy's law. When gravity is neglected, it writes

$$
q=\phi v=-\frac{K_{v}}{\mu} \cdot \nabla P
$$

where $\nabla P$ is the pressure gradient vector and $\boldsymbol{q}$ the filtration or Darcy's velocity, which is related to the pore (interstitial) average velocity $\boldsymbol{v}$ and the porosity $\phi$.

\section{Sliding models}

When $K_{n} \sim 1$, an interaction between Knudsen and viscous flow produces a pattern referred to as sliding flow.

Dust gas model. In the dust gas model, ${ }^{16}$ the general flux equation is

$$
J_{\text {slid }}=-\frac{1}{R T}\left[K_{m} \bar{v}+\frac{P_{a v g} K_{v}}{\mu}\right] \nabla P
$$

where $R$ is the ideal gas constant and $P_{\text {avg }}$ the average of pressures at both sides of the considered domain. From a mathematical point of view, equation (5) is a 
sum of equations (2) and (3) substituting the molecular density $\tilde{n}=n / V$ using the ideal gas law (where $n$ is the number of moles in the considered volume $V$ ). With this formulation, the viscous permeability can be extracted from the overall flow even if it combines viscous and Knudsen flows.

Klinkenberg sliding effect. The Klinkenberg sliding effect is observed and promoted on gas transport through finegrained low-permeability porous media, such as soils, with small pore size. ${ }^{18}$ At low rates, the effective permeability $K_{g}$ measured with $\mathrm{N}_{2}$ gas (calculated using Darcy's law) will be higher than the viscous permeability $K_{\infty}$ measured with brine. Klinkenberg explains changes of permeability under different pressures by the slippage of gases along the pore walls, since gas does not adhere to the pore walls as liquid does. The effective gas permeability $K_{g}$ depending on gas pressure is given by

$$
K_{g}=K_{\infty}\left(1+\frac{b}{P}\right)
$$

where $K_{\infty}$ is the intrinsic viscous permeability, which is considered to be the absolute permeability under very large gas pressure at which condition the Klinkenberg effect is negligible. The Klinkenberg factor $b$ depends on the mean free path of the gas molecules and the microstructure of the pores. Jones and Owens ${ }^{19}$ fitted it to

$$
b=\alpha_{k} K_{\infty}^{-0.36}
$$

where $\alpha_{k}$ is the Klinkenberg effect coefficient, which is fitted to 0.251 based on the experimental data of 100 rock samples ranging in permeability from $10^{-17}$ to $10^{-12} \mathrm{~m}^{2} .^{20}$

\section{Review on permeability measurements using gas}

\section{Porous material}

Because of low permeabilities of soils, rocks, ceramics and membranes, many set-ups have been built using pressures greater than atmospheric pressure. ${ }^{12,13,21}$ Measurements can be stationary (e.g. constant flow rate) or transient (e.g. raising or dropping pressure). The stationary flow measurement is the simplest way to calculate permeability because the flow rate is directly measured. Transient flow measurement has the advantage of requiring simple equipments and no flow rate meter. The permeabilities obtained by a pressure-decay method and a stationary method show an agreement for dense ceramics. ${ }^{13}$ Unsteady gas flow is used extensively to determine permeability of soil in field tests ${ }^{14}$ or in laboratory tests with dropping ${ }^{13,22}$ or raising pressure methods (DPMs or RPMs). ${ }^{15}$

\section{Fibrous material}

The literature of fibrous permeability measurement using gas as fluid is rich. Because of the nature of the process, the chemical vapour infiltration requires a good knowledge of gas permeability. Starr and Hablutzel ${ }^{5}$ proposed a 1D steady-state gas flow technique using helium gas. Permeability is extracted from Darcy's law integrated for an incompressible fluid and using a differential pressure gauge and a flow meter. Later, several researchers have modified and/or improved the steady gas flow technique to measure fibrous reinforcement permeability. ${ }^{7,8,10}$ Those authors build 1D or 2D (annular) benches and measured permeability with various gases: nitrogen, helium or air. The system of equations to model the flow included Darcy's law and mass conservation (compressible fluid). The permeability is either obtained from an analytical solution of the system of equation or minimizing a difference between experimental and simulation results by an inverse method. The main issue of these techniques rely on the fact that depending on the level of permeability to measure, the flow meter and pressure transducers have to be changed to comply with the pressure and flow rate levels to match low Reynolds numbers during the experiment. Also, techniques using annular domains limit their use to in-plane isotropic media.

One way to overcome the issue of measuring flow rates is to use transient techniques as presented by Kim and Daniel ${ }^{11}$ and Sequeira Tavares et al. ${ }^{9}$ The transient evolution of the pressure levels at different locations during the experiments are used to extract a value of permeability. Some assumptions are done to simplify the system of equations and obtain analytical solutions of pressure profiles at specific locations in the domain with respect to time.

\section{Permeability measurement of fibrous material using vacuum}

\section{Experimental apparatus and materials}

The equipment designed to measure air permeability of fibrous preforms by 1D flow is shown in Figure 1. The preform is inserted between a set of top $(30 \mathrm{~mm}$ thick PMMA; poly (methyl methacrylate)) and bottom (20 mm-thick metallic) plates, sealed with a rubber o-ring seal. The outlet and inlet are, respectively, connected to a vacuum pump and the atmosphere, and 


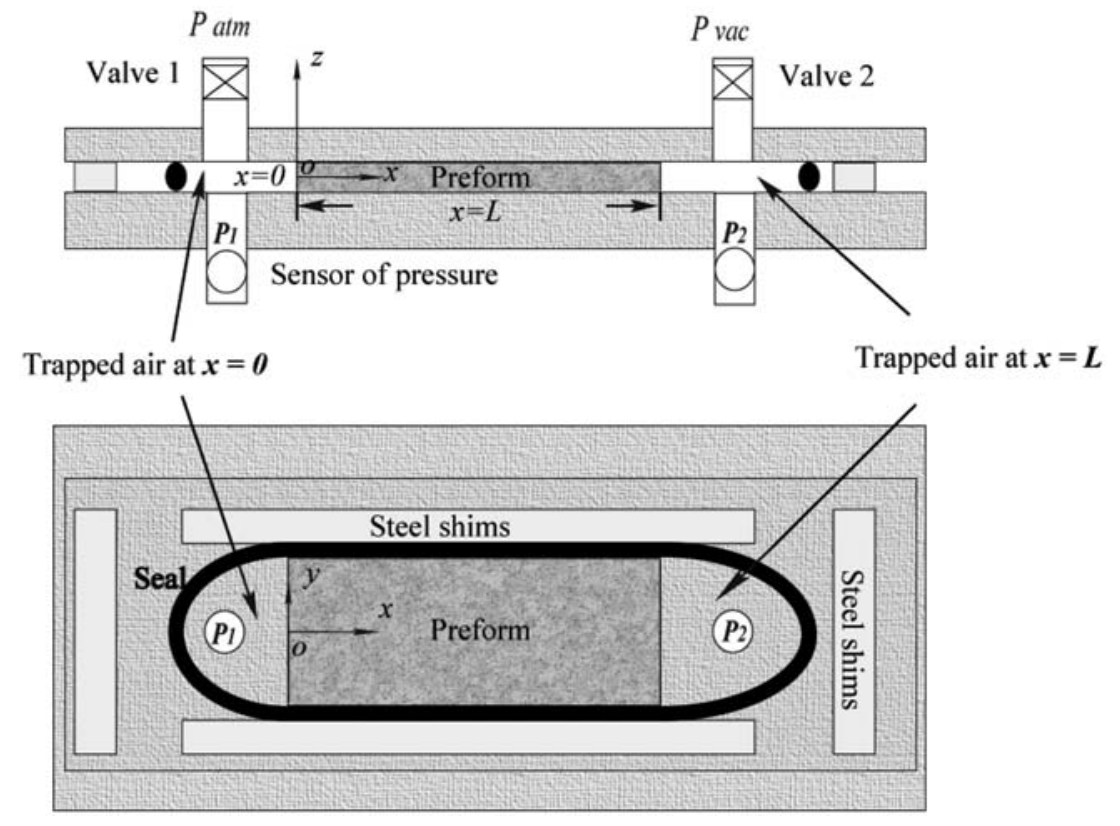

Figure I. ID experimental apparatus for air permeability measurement.

controlled by valves. Four pairs of $50 \mathrm{~mm}$-thick steel stiffeners are screwed so as to compact the preform and to force the PMMA and steel platens to be in contact with the steel shims, with a deviation on thickness below $0.05 \mathrm{~mm}$ during the test. The steal shims are positioned to secure a tight contact between the fabric edge and seal. Hence, the gap between the fabric and seal is the same magnitude of pore size, and racetracking is negligible. The same set-up has also been used for the permeability measurements using liquid flow; no racetracking with liquid was observed at the boundaries.

The pressures $P_{1}$ and $P_{2}$ are monitored by pressure gauges (Kistler 4260A Series, $0-1$ bar absolute, $1 \%$ accuracy) at room temperature and recorded with the help of a data acquisition system. The laboratory vacuum pump delivers a vacuum level of $3 \times 10^{3} \mathrm{~Pa}$ (absolute). Carbon-based reinforcement combined with representative architectures (woven, unidirectional and bidirectional) have been selected. The main features of the materials and the preform configurations are given in Table 1. The average size of the samples were $250 \mathrm{~mm}$ in length and $150 \mathrm{~mm}$ in width. Each permeability for a given fibre volume fraction has been measured between 2 and 5 times with up to four types of loading pressures.

\section{Transient flow tests}

Two types of 1D transient flows are studied: the DPM or RPM. To create a flow using the DPM, the test
Table I. References of the materials tested in the study.

\begin{tabular}{llll}
\hline Label & CTW & CBD & CUD \\
\hline Architecture & Twill-weave & Bidirectional & Unidirectional \\
& $2 \times 2$ & stitched & \\
Fibre type & Carbon & Carbon & Carbon \\
Areal weight $\left(\mathrm{g} / \mathrm{m}^{2}\right)$ & 285 & 548 & 150 \\
Manufacturer & Hexcel & Saertex & Hexcel \\
Reference & $\mathrm{G} 986$ & Confidential & Hexforce 43I5I \\
Stacking & {$[0]_{6}$} & {$[+/-45]_{4}$} & {$[0]_{15}$ and $[90]_{15}$} \\
$V_{f}$ range & {$[0.44,0.55]$} & {$[0.52,0.59]$} & {$[0.55,0.67]$} \\
\hline
\end{tabular}

CTW: carbon twill-weave; CBD: carbon bidirectional; and CUD: carbon unidirectional.

begins by setting the initial pressure within the cavity and the sample to the atmospheric pressure $P_{a}$. This is obtained by closing valve 2 and opening valve 1 until the values of $P_{1}$ and $P_{2}$ become equal (Figure 1). Then, valve 1 is closed and valve 2 is opened to let the vacuum in (or the gas out). For a RPM test, the experiment begins with an initial vacuum within the preform and cavity; then keeping valve 2 closed, valve 1 is opened to let the gas in.

\section{Flow regime}

In this section, the flow regimes induced by RPM and DPM tests are evaluated. Viscous flow regimes (laminar or turbulent) are governed by the Reynolds number $R e$. 


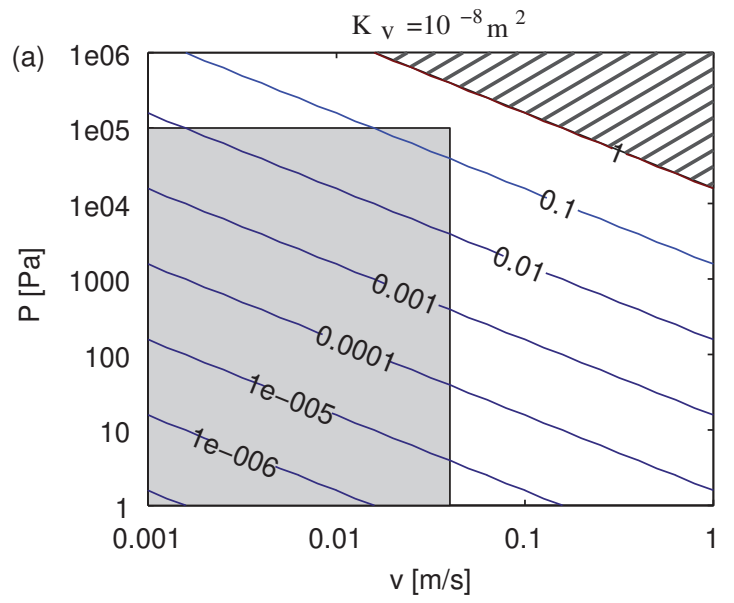

Figure 2. Reynolds number related to pressure and velocity.

In the case of porous or fibrous media, a modified $R e_{\phi}$ can be defined

$$
R e_{\phi}=\frac{\rho v l_{\phi}}{\mu}
$$

where $l_{\phi}$ is a characteristic pore size, $\mu$ the viscosity, $v$ the gas velocity and $\rho$ the gas density. It is accepted that $\sqrt{K_{v}}$ is an appropriate estimate of $l_{\phi}$. Typical fibre preforms of interest in structural composites exhibit an in-plane permeability in a range $10^{-13}$ to $10^{-8} \mathrm{~m}^{2}$. Figure 2 shows the $R e_{\phi}$ field for given gas velocities $v$ and pressures $P$, in the two extreme cases of permeabilities of interest $10^{-13}$ and $10^{-8} \mathrm{~m}^{2}$. Density is evaluated from the pressure. Temperature is $20^{\circ} \mathrm{C}$ and sets the viscosity. The dark regions in Figure 2 show the regions of interest for the materials and test conditions of this study. Therefore, for this type of flow, Figure 2 shows that $R e_{\phi}<1$ for a maximum absolute pressure of $10^{5} \mathrm{~Pa}$. The flow remains laminar and therefore governed by Darcy's law. The non-linearities involved with higher $R e_{\phi}$ that would require the use of Forchheimer's relationship are not of concern for most of fibrous preforms of interest in this study. Since perfect vacuum does not exist, partial vacuum (or pressure) is of concern in this study. The fibrous medium is therefore saturated with gas molecules. When the pressure increases or decreases, gas molecules are added or removed. The gas density varies along the sample due to the motion of molecules. The notion of impregnated/not impregnated region that define a fluid flow front does not exist with gas.

\section{Thermal analysis}

The flow of gas in the fibrous preforms can be compressed or expanded depending on the type of experiments that will be performed. Such physical change implies a temperature variation that has to be

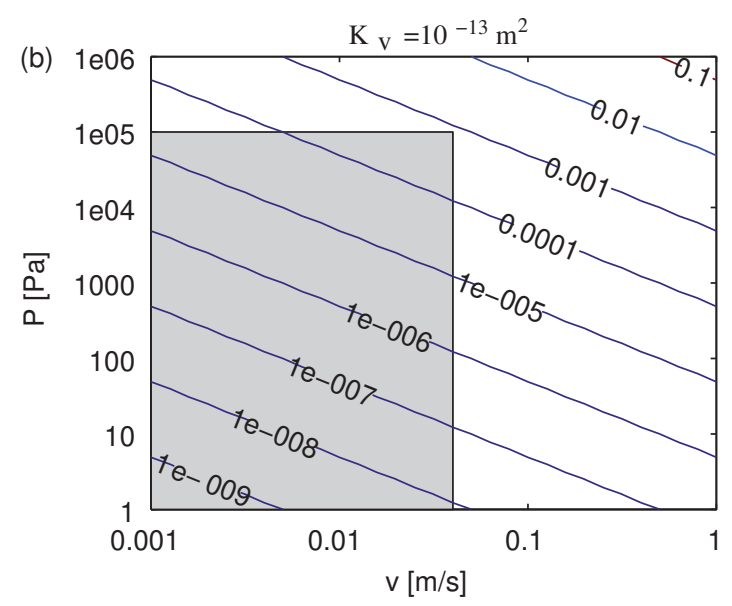

estimated. This possible temperature change is important to be checked because, first, the viscosity of gases is temperature dependent (Sutherland's relationship)

$$
\mu_{a}=\mu_{0}\left(\frac{T_{0}+C}{T+C}\right)\left(\frac{T}{T_{0}}\right)^{3 / 2}
$$

where e.g. for air, $\mu_{0}=1.81 \times 10^{-5} \mathrm{~Pa} \cdot \mathrm{s}, T_{0}=293 \mathrm{~K}$ and $C=117 \mathrm{~K}$. Second, the temperature influences the state equation of an ideal gas

$$
P V=n R T
$$

where $n$ is the number of moles and $R$ the ideal gas constant $(8.314 \mathrm{~J} /(\mathrm{mol} \cdot \mathrm{K}))$.

Thermodynamics can be used to predict the approximate temperature variation during the test. The first law for open systems gives, ${ }^{23}$

$$
\mathrm{dU}=\delta \mathrm{Q}+\mathrm{dH}_{\text {in }}-\mathrm{dH}_{\text {out }}
$$

where $\mathrm{d} U$ is the differential change in internal energy, $\delta Q$ the infinitesimal amount of heat supplied to the system, $\mathrm{d} H_{\text {in }}$ and $\mathrm{d} H_{\text {out }}$ the enthalpy entering and leaving the system, respectively. For instance, in the case of a RPM test where the cavity is initially emptied (vacuumed), one valve is opened to let gas at atmospheric pressure in. The second valve remains closed (no gas exits the system), therefore $\mathrm{d} H_{\text {out }}=0$. For an ideal gas, the internal energy $U$, enthalpy $H$ and heat $Q$ are related to temperature $T$ as

$$
\begin{aligned}
\mathrm{d} U & =\hat{C}_{v} R \cdot \mathrm{d}(n T) \quad \mathrm{d} H_{\text {in }}=\left(1+\hat{C}_{v}\right) R T \cdot \mathrm{d} n \\
\delta Q & =h_{c} A_{1}\left(T_{0}-T\right) \mathrm{d} t
\end{aligned}
$$

where $\hat{C}_{v}$ is the dimensionless specific heat capacity at constant volume (3/2 for monoatomic gas, $5 / 2$ for diatomic gas and 3 for more complex molecules, 
$A_{1}$ the surface of air in contact with the two plates of the cavity, $h_{c}$ the heat transfer coefficient, from 10 to $100 \mathrm{~W} /\left(\mathrm{m}^{2} \cdot \mathrm{K}\right)$ for air and $T_{0}$ the environment temperature. Substituting equations (10) and (12) into equation (11), leads to

$$
\left(1+\hat{C}_{v}\right) \frac{P H}{2 T} \frac{\mathrm{d} T}{\mathrm{~d} t}+h_{c} T-\left(\frac{H}{2} \frac{\mathrm{d} P}{\mathrm{~d} t}+h_{c} T_{0}\right)=0
$$

where $H=2 V / A_{1}$ is the thickness of the cavity and $\hat{C}_{v}=5 / 2$. Equation (13) is a first-order differential equation which can be solved with the variable-order method. Representative test pressures (Figure 3(a)) are used as inputs and the resulting temperature change is less than 3\%, as shown in Figure 3(b). Therefore, the induced viscosity variation is negligible. Similarly, for the RPM experiments, the temperature and viscosity changes are even lower.

\section{Governing equations}

It has been shown in the previous sections that the flow induced in the RPM and DPM tests of this study is laminar and can be considered as isothermal. The three unknowns of a compressible flow problem are density, pressure and velocity. Therefore, three equations are necessary: (a) mass conservation, (b) equation of state and (c) momentum equation.

For a compressible fluid, the mass conservation equation is given in the Eulerian frame by

$$
\frac{\partial \rho}{\partial t}+\nabla \cdot(\rho v)=0
$$

The equation of state of an ideal gas is given by equation (10). For the fibrous materials of interest in structural composite manufacturing, two distinct momentum equations for viscous and sliding regime (Knudsen flow can be simulated using sliding model

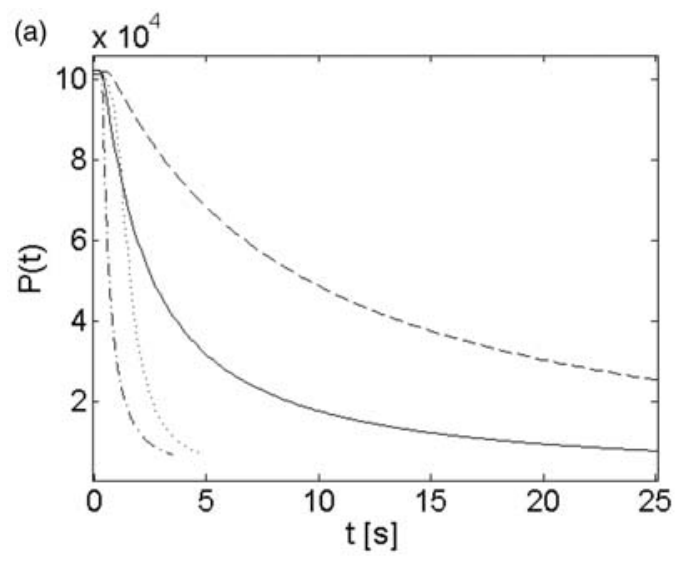

by setting $K_{v}=0$ ) have to be considered. For a flow in the viscous regime with neglected gravity, the governing momentum equation is obtained by substituting equations (4) and (10), respectively, Darcy's law and ideal gas law, into equation (14)

$$
\frac{\partial P}{\partial t}=\frac{1}{\phi \mu} \nabla \cdot P K_{v} \nabla P
$$

When the flow is not governed by the viscous effects, inverse method based on Darcy's law (equation (15)) does not return the viscous permeability $K_{v}$ but the average efficient permeability $\bar{K}_{g}$. In order to obtain the correct viscous permeability, sliding model (equation (5)) can be used with equations (10) and (14). The governing momentum equation in this case becomes for 1D flow

$$
\frac{\partial P}{\partial t}-\frac{1}{\phi} \frac{\partial}{\partial x}\left[\left(K_{m} \bar{v}+\frac{K_{v} P}{\mu}\right) \cdot \frac{\partial P}{\partial x}\right]=0
$$

\section{Boundary and initial conditions}

Since air is compressible, the air trapped between the valve and the edges of the preform, as shown in Figure 1, may cause fluxes at the boundaries when pressure changes. The gas present in pipes and tubing outside the valves are not affecting the test. Assuming a quasistatic flow, the trapped air shares the same values of pressure, density and temperature. For the points $x=0$ and $x=L$, the mass conservation equation (14) gives

$$
\begin{array}{ll}
\frac{\partial \rho}{\partial t} V+\rho A q=0 & x=0 \\
\frac{\partial \rho}{\partial t} V-\rho A q=0 & x=L
\end{array}
$$

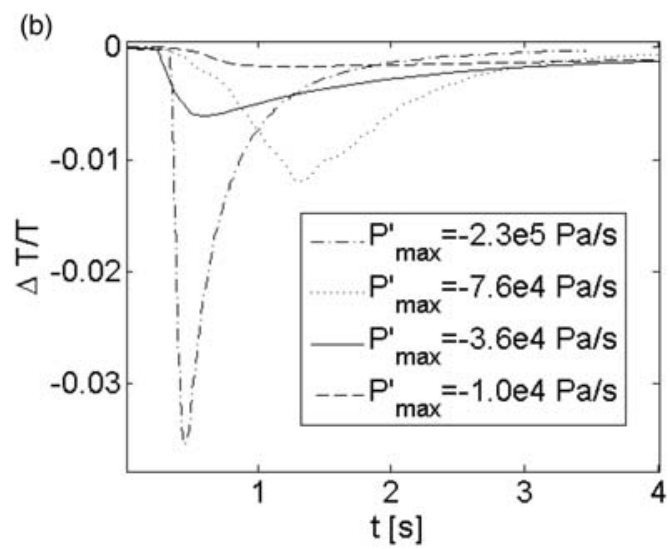

Figure 3. Pressure (a) and temperature (b) variations calculated during DPM tests $\left(P^{\prime}=\partial P / \partial t\right)$. DPM: dropping pressure method. 
where $V$ is the volume of trapped air, $A$ the cross-sectional area normal to the gas flow and $L$ the length of the fabric sample. Combining equation (17) with the ideal gas law (equation (10)) and Darcy's law (equation (4)), with $K_{g}$ used instead of $K_{v}$ ), the boundary conditions can be obtained in terms of pressure $P$

$$
\begin{array}{ll}
\frac{K_{g}}{\mu} P \frac{\partial P}{\partial x}-\frac{\partial P}{\partial t} \frac{V}{A}=0 & x=0 \\
\frac{K_{g}}{\mu} P \frac{\partial P}{\partial x}+\frac{\partial P}{\partial t} \frac{V}{A}=0 & x=L
\end{array}
$$

where the volume-to-area ratio, $V / A$, is the dominant parameter. For a set of experimental pressures $P_{1}$ and $P_{2}$, the permeability obtained by inverse method could change remarkably with different volume-to-area ratios. The variation of the calculated permeability, if the trapped air is taken into account or not can be estimated as

$$
\frac{K_{g}}{K_{0}}=1+2 \frac{V}{A L \phi}
$$

where $K_{0}$ is the permeability obtained by inverse method with unmodified boundary conditions (i.e. neglecting trapped air). As for initial conditions, for RPM tests, the pressure is set to $P(x, t=0)=P_{\text {vac }}$ (pressure obtained with vacuum pump) and for DPM tests $P(x$, $t=0)=P_{a}$, where $P_{a}$ is the atmospheric pressure.

\section{Inverse method}

The simulation is based on solving the set of fundamental equations for $P$ (equation (15) or (16)) with proper boundary (equation (18)) and initial conditions.
The room temperature $T_{o}$ is recorded before each experiment. The gas viscosity $\mu$ is calculated from that temperature with equation (9). Pressures $P_{1}(t)$ and $P_{2}(t)$ are recorded during experiments. The porosity $\phi$ of the sample is provided by the sample thickness, the number of plies and the areal weight (Table 1).

Then permeability $K_{v}$ (or $\left[K_{v}, K_{m}\right]$ in sliding model) is estimated by inverse method: the quadratic residual $\epsilon$ (equation (20)) between experimental and simulated results is minimized to obtain the best-fitted permeability. For RPM tests, $P_{2}(t)$ is compared, and simulated results are obtained from equation (17b) at $x=L$ under the prescribed pressure $P_{1}(t)$ at $x=0$. In the case of DPM tests, the experimental and simulated pressures $P_{1}(t)$ are matched for given pressure $P_{2}(t)$ at $x=L$ and equation (17a) at $x=0$. The fitting error writes, for RPM tests

$$
\epsilon=\frac{1}{P_{a}} \sqrt{\frac{1}{N} \sum_{i=1}^{N}\left(P_{2 \exp }^{i}-P_{2 \operatorname{sim}}^{i}\right)^{2}}
$$

where $P_{2 \exp }$ is the experimental pressure and $P_{2 \operatorname{sim}}$ the simulated pressure at the boundary $2, N$ the number of data points $i$ and $P_{a}$ the atmospheric pressure.

\section{Results}

\section{Evaluation of measurements for Darcy's flow}

DPM and RPM have been applied to CTW preforms at $48.6 \%$ fibre volume fraction (Figure 4). In those cases, $K_{n}<1$, therefore simulations of flow based on Darcy's law fits well the experimental data.
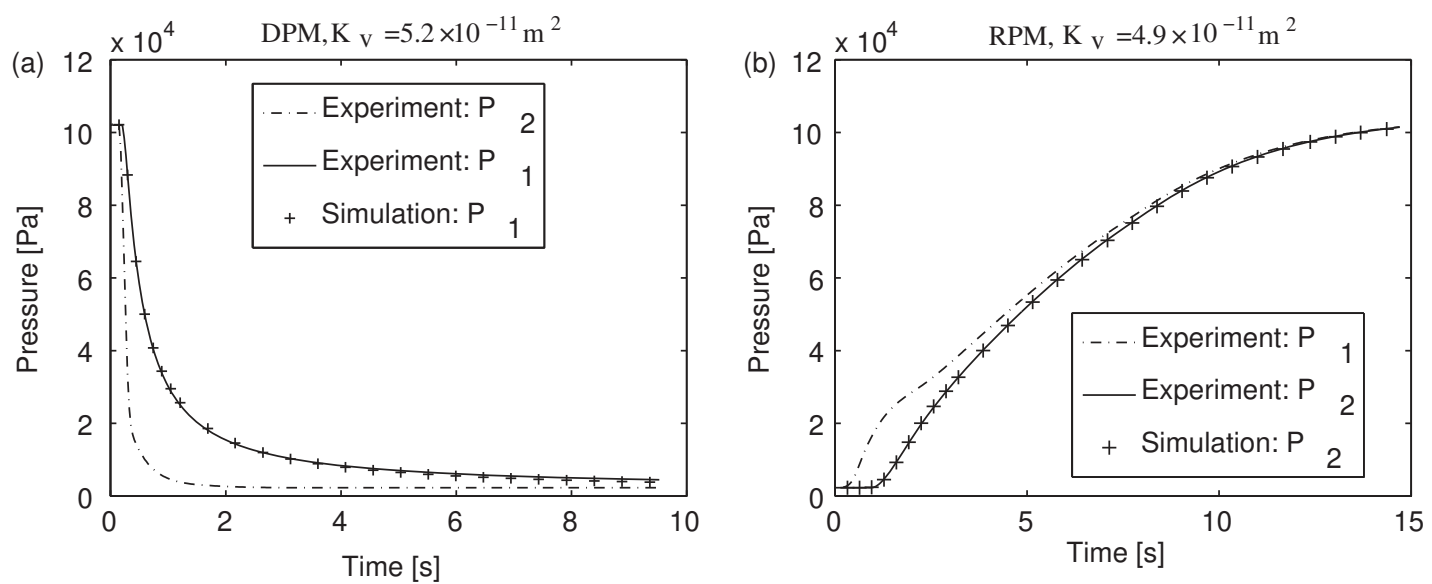

Figure 4. Comparison between experimental and simulated pressures $P_{2}$ for CTW preforms at $48.6 \%$ fibre volume fraction. DPM: dropping pressure method; RPM: raising pressure method; and CTW: carbon twill-weave. 
The air transient measurements of the CTW reinforcements are carried out for various volume fractions. Results show that permeability has a similar trend as those extracted from liquid injection and compression tests ${ }^{3}$ (Figure 5). Also, the use or nitrogen $\left(\mathrm{N}_{2}\right)$ instead of air shows no major change in the results.

\section{Sliding effect in air transport through porous media}

It has been seen in 'Fundamentals on gas flow through porous media' section that gas flow in porous media is governed by the $K_{n}$ number. Using equation (1) and considering that $l \simeq \sqrt{K_{v}}$, the $K_{n}$ number field can be plotted in the absolute pressure and permeability domain (Figure 6). The domain is chosen based on the fibrous materials of interest $\left(K_{v} \in\left[10^{-15} ; 10^{-6}\right] \mathrm{m}^{2}\right.$ and $\left.P \in\left[10^{0} ; 10^{6}\right] \mathrm{Pa}\right)$. For instance, as it can be seen from Figure 6 that for CTW preforms, $K_{v} \in\left[10^{-11}\right.$; $\left.10^{-9}\right] \mathrm{m}^{2}$, the pressure drops from $10^{5}$ to $10^{3} \mathrm{~Pa}$ in a DPM test and the flow is mostly viscous and governed by Darcy's law. For CUD with $K_{v} \in\left[10^{-14} ; 10^{-12}\right] \mathrm{m}^{2}$, the flow is mostly governed by the sliding and Knudsen regimes. Consequently, equation (16) is applied in this case and a better fitting could be obtained, relatively to using Darcy's law (viscous flow), as shown in Figure 7. The sliding effect can be explained by Knudsen flow ${ }^{16}$ or Klinkenberg effect. ${ }^{18}$ Although the two models come from different theories, they represent the same phenomenon, and hence, permeability can be expressed equivalently with equations (5) and (6), $\left[K_{m}, K_{v}\right]$ and $\left[b, K_{\infty}\right]$ related by

$$
K_{\infty}=K_{v}, \quad b=\frac{K_{m}}{K_{v}} \bar{v} \mu
$$

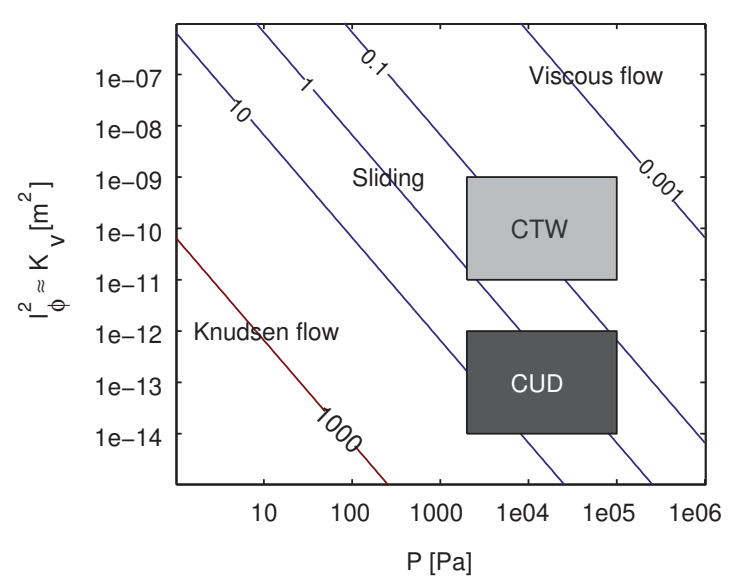

Figure 6. Knudsen number field in the absolute pressure and permeability domain.

CTW: carbon twill-weave; CUD: carbon unidirectional.

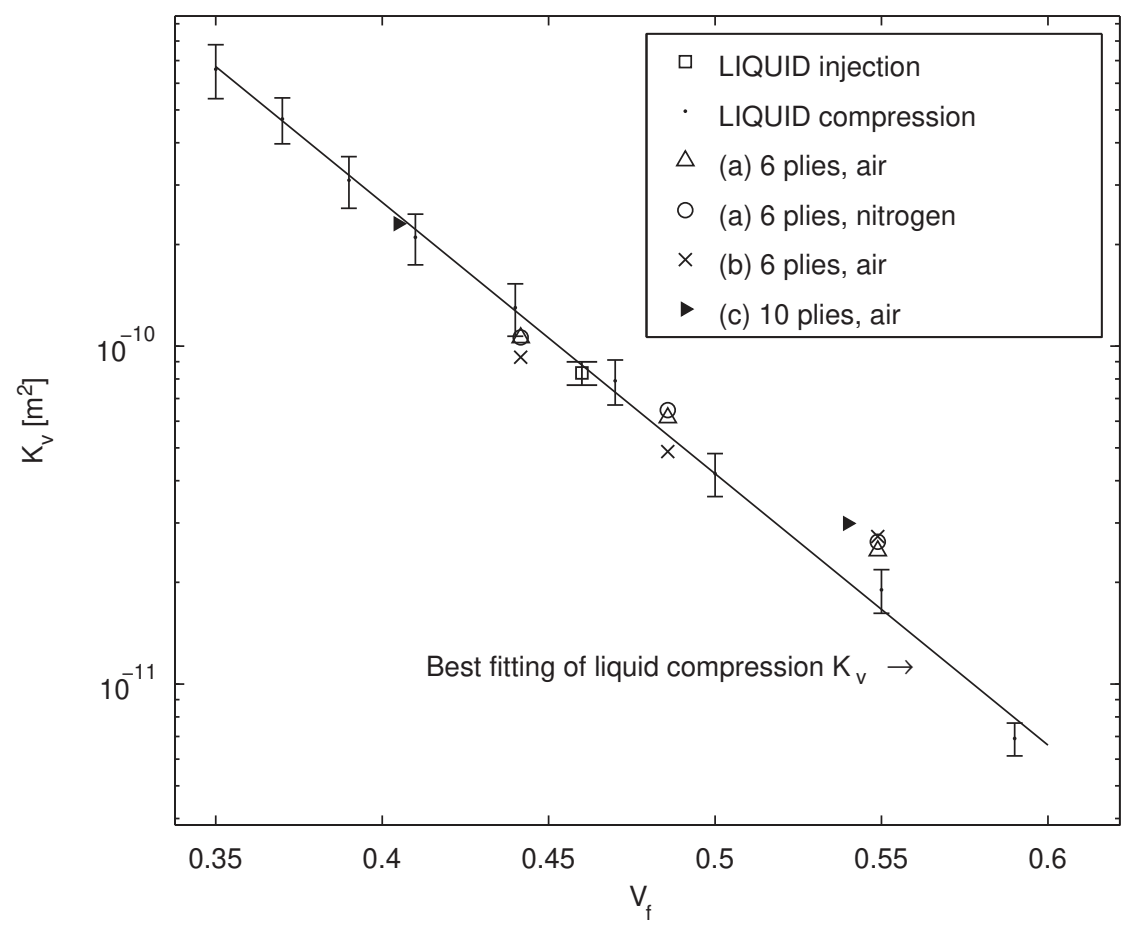

Figure 5. Comparison of permeability $K_{v}$ obtained by transient air flow (back-calculated based on Darcy's law: $\epsilon<0.7 \%, \bar{K}_{g} \approx K_{v}$ ), liquid compression and liquid unidirectional injection measurement methods on CTW for different volume fractions. ${ }^{3}$

CTW: carbon twill-weave. 
The previous section has shown that the deviation of transient gas measurement compared with liquid compression is less than $25 \%$ for the cases that do not display sliding. When sliding occurs, the apparent permeability measured using gas can be slightly higher than the one using liquid, but the viscous permeability back-calculated using a correct sliding model shows a coincidence between liquid and gas measurements.

For a tube, theoretical values of $K_{v}$ and $K_{m}$ predicted by Mason and Malinauskas ${ }^{16}$ are available, from which $b$ can be deduced

$$
b=\frac{32 \sqrt{2}}{3} K_{v}^{-0.5}
$$

A comparison of $b$ related to $K_{v}$ for different structures shows that the way $b$ depends on $K_{v}$ is determined by the local microstructure (Figure 8: tube by Mason's theoretical prediction, ${ }^{16}$ granular media revealed from experiments on soils and rocks by Jones and Owens, ${ }^{19}$ and fibrous media from experiments of this study).
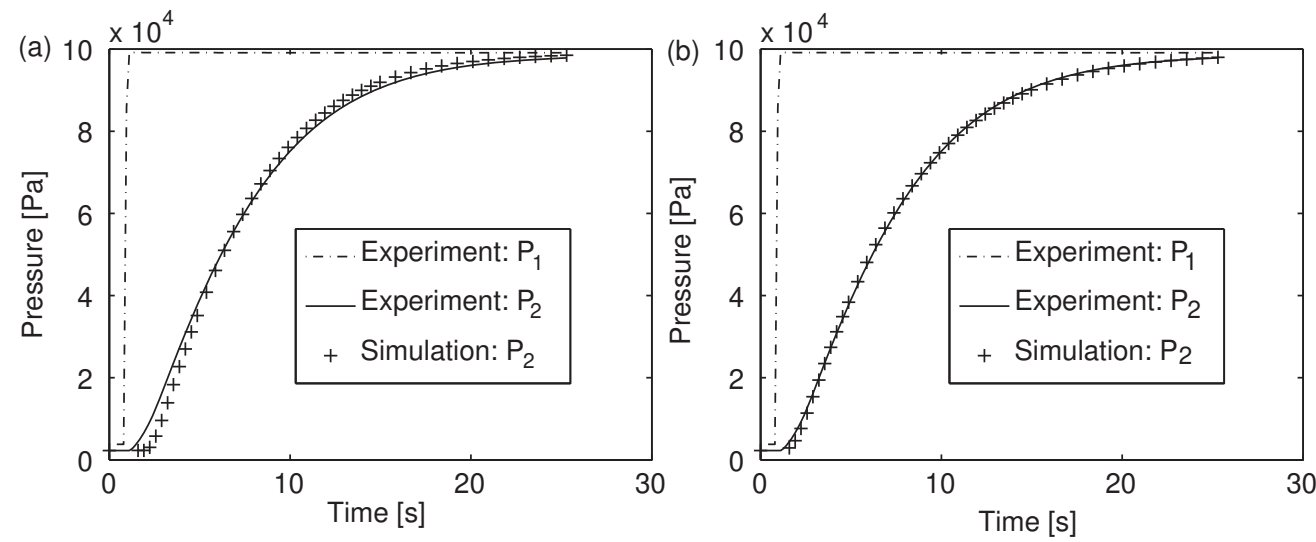

Darcy's law, $\varepsilon=22 \%, \overline{\mathrm{K}} \mathrm{g}=1.7 \times 10^{-12} \mathrm{~m}^{2}$

Sliding model, $\varepsilon=0.6 \%: \mathrm{K}_{\infty}=\mathrm{K}_{v}=9.3 \times$
$10^{-13} \mathrm{~m}^{2} ; \mathrm{K}_{\mathrm{m}}=5.5 \times 10^{-6} \mathrm{~m}, \mathrm{~b}=0.5 \times 10^{5} \mathrm{~Pa}$.

Figure 7. Comparison between experimental and simulation pressures $P_{2}$ using Darcy's law (a) and sliding model (b), in RPM for CUD preforms in the direction parallel to the fibers at $60.8 \%$ fibre volume fraction.

RPM: raised pressure method; CUD: carbon unidirectional.

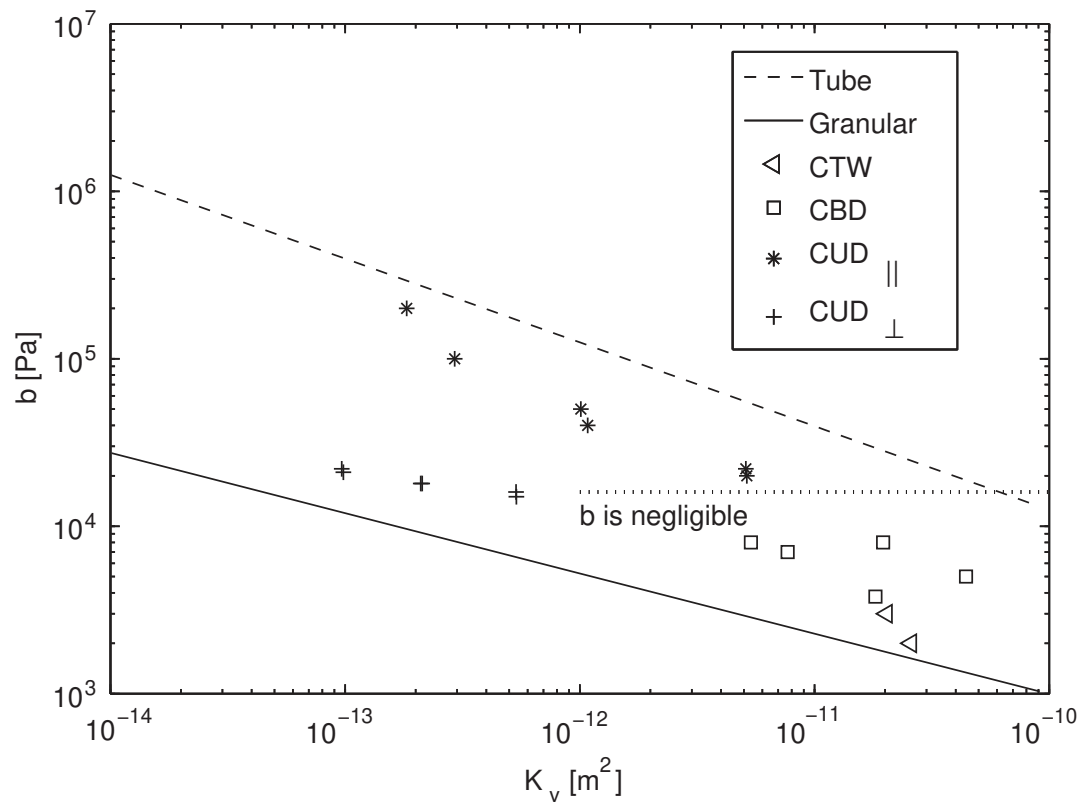

Figure 8. Comparison of the coefficient $b$ related to $K_{v}$ for different structures.

CTW: carbon twill-weave; CBD: carbon bidirectional; and CUD: carbon unidirectional. 
The Klinkenberg coefficients $b$ obtained for the fibrous materials of the study lie between the two extreme cases of tubular and granular microstructures; for CUD (CUD in the direction parallel to fibres), the relationship of $b$ and $K_{v}$ is close to the one for tubular microstructures.

Figure 8 also shows flow regimes: viscous flow dominates when $b$ is smaller than $3 \times 10^{3} \mathrm{~Pa}$ and becomes undetectable; Knudsen flow dominates when $b$ is close to $1 \times 10^{6} \mathrm{~Pa}$ and $K_{v}$ is undetectable. According to Klinkenberg's model (equation (6)), once $b$ is estimated, flow regime can be determined by a comparison between $b$ and experimental pressure.

\section{Conclusion}

A methodology to measure fabric in-plane permeability of fibrous reinforcements using a transient air flow has been described. The method, based on the simple measurement of gas pressure throughout the transient flow, is convenient, clean and fast, avoids usage of a gas flow meter and offers a way to study the air transport within fibrous media. Beside the cleanliness/rapidity and the limited number of sensors, a thorough gas flow (at vacuum levels) regime study has been provided, i.e. laminar vs. turbulent regimes, sliding vs. no sliding regimes and thermal effects during the experiment. Therefore, this study gives specific criteria of regimes when Darcian or sliding model can be used. For each range of fibrous media, one can choose the appropriate set of equations to solve when performing gas flow measurement.

The former studies also made assumptions like: negligible gas compressibility ${ }^{5}$ or low Reynolds number ${ }^{7}$ without actually verifying them. Gas compressibility, has to be considered under a high-pressure gradient. ${ }^{12}$ Since pressure varies from $3 \times 10^{3}$ to $10^{5} \mathrm{~Pa}$, compressibility becomes very important.

Transient gas flow has been used to detect the defects of preforms and determine permeability, ${ }^{9,11}$ although no author mentioned the risks and consequences of eventual trapped air at the boundaries. Fundamental equations and boundary conditions for gas flow in fibrous media have been proposed. At the boundary where gas flow is cut off by a closed valve, a slight flux exists due to compressibility of the gas trapped between the sample and valve and this changes the overall gas flow field of the sample, leading to a significantly underestimated permeability if proper boundary condition is not implemented. To estimate the correction to apply on the permeability measured by ignoring gas compressibility at the boundaries, an empirical relationship related to trapped gas volume and crosssectional area of gas flow is proposed. This has an advantage if one wants to use air instead of fluid on a conventional liquid injection bench.
Darcy's law (viscous flow) can be applied to gas flow through fabric with large pore size and high pore pressure. For smaller pore size and lower pore pressure, gas molecules slide on pore walls (Figure 6). The sliding effect, also called Klinkenberg effect, ${ }^{18,20,24}$ reveals a dependence of apparent permeability on pressure in porous media. In rocks and soils, the Klinkenberg effect is considered important when permeability is lower than $10^{-18} \mathrm{~m}^{2},{ }^{24}$ while in this study, the Klinkenberg effect is noticeable although the permeability is much higher. This difference is due to the fact that high pressures are used in rocks and soil permeability measurements. To explain this, the Knudsen flow theory ${ }^{16}$ is introduced into the fibrous media. This model provides a more thorough view into the sliding flow regime, evaluating sliding effect with Knudsen number. The results of viscous permeability measured using gas match well the permeability measured with liquid compression and injection techniques. Also, Klinkenberg coefficient $b$ of fibrous media is related to viscous permeability $K_{v}$ with a same trend as tubular and granular media.

\section{Funding}

The authors thank the European Union (Seventh Framework Programme) for funding this study within the INFUCOMP project (grant reference: 233926).

\section{Conflict of interest}

None declared.

\section{References}

1. Arbter R, Beraud J, Binetruy C, et al. Experimental determination of the permeability of textiles: a benchmark exercise. Composites Part A: Appl Sci Manuf 2011; 42: 1157-1168.

2. Buntain MJ and Bickerton S. Compression flow permeability measurement: a continuous technique. Composites Part A: Appl Sci Manuf 2003; 34(5): 445-457.

3. Comas-Cardona S, Binetruy $\mathrm{C}$ and Krawczak $\mathrm{P}$. Unidirectional compression of fibre reinforcements. Part 2: a continuous permeability tensor measurement. Compos Sci Technol 2006; 67(3-4): 638-645.

4. Ouagne P and Breard J. Continuous transverse permeability of fibrous media. Composites Part A: Appl Sci Manuf 2010; 41: 22-28.

5. Starr TL and Hablutzel N. Measurement of gas transport through fibre preforms and densified composites for chemical vapor infiltration. $J$ Am Ceram Soc 1998; 81(5): 1298-1304.

6. Ding L, Shih C, Liang Z, et al. In situ measurement and monitoring of whole-field permeability profile of fibre preform for liquid composite molding processes. Composites Part A: Appl Sci Manuf 2003; 34: 779-789. 
7. Feser J, Prasad A and Advani S. Experimental characterization of in-plane permeability of gas diffusion layers. J Power Sources 2006; 162(2): 1226-1231.

8. Pomeroy R, Grove S, Summerscales J, et al. Measurement of permeability of continuous filament mat glass-fibre reinforcements by saturated radial airflow. Composites Part A: Appl Sci Manuf 2007; 38(5): 1439-1443.

9. Sequeira Tavares S, Michaud V and Manson J-A. Through thickness air permeability of prepregs during cure. Composites Part A: Appl Sci Manuf 2009; 40: $1587-1596$.

10. Um M, Daniel IM and Childs BW. A gas flow method for determination of in-plane permeability of fibre preforms. Polym Compos 2001; 22(1): 47-56.

11. Kim SK and Daniel IM. Transient gas flow technique for inspection of fibre preforms in resin transfer molding. Composites Part A: Appl Sci Manuf 2005; 36(12): 1694-1699.

12. Groenewoud HV. Methods and apparatus for measuring air permeability of the soil. Soil Sci 1968; 106(4): 275-279.

13. Innocentini MDM, Rizzi AC, Nascimento LA, et al. The pressure-decay technique for air permeability evaluation of dense refractory ceramics. Cem Concr Res 2004; 34(2): 293-298.

14. McWhorter DB. Unsteady radial flow of gas in the vadose zone. J Contam Hydrol 1990; 5(3): 297-314.

15. Cho JS and Ellett D. Soil air permeability measurement with a transient pressure buildup method. Hazard Waste Hazard Mater 1995; 12(4): 365-371.
16. Mason EA and Malinauskas AP. Gas transport in porous media: the dusty-gas model. Chemical engineering monographs 17. Amsterdam: Elsevier Scientific, 1983.

17. Knudsen M. The kinetic theory of gases. London: Methuen and Co., Ltd, 1934.

18. Klinkenberg L. The permeability of porous media to liquids and gases. Drilling and production practice. American Petroleum Institute, 1941, pp.200-213.

19. Jones $\mathrm{F}$ and Owens W. A laboratory study of lowpermeability gas sands. J Pet Technol 1980; 32(9): 1631-1640.

20. Wu Y, Pruess $\mathrm{K}$ and Persoff P. Gas flow in porous media with Klinkenberg effects. Transp Porous Media 1998; 32(1): 117-137.

21. Iversen BV, Schjonning P, Poulsen TG, et al. In situ, onsite and laboratory measurements of soil air permeability: Boundary conditions and measurement scale. Soil Sci 2001; 166(2): 97-106.

22. Springer DS, Loaiciga HA, Cullen SJ, et al. Air permeability of porous materials under controlled laboratory conditions. Ground Water 1998; 36(4): 558-565.

23. Cengel YA and Ghajar AJ. Heat and mass transfer: fundamentals and applications. New York: McGraw-Hill, 2011.

24. Tanikawa $\mathrm{W}$ and Shimamoto T. Comparison of Klinkenberg-corrected gas permeability and water permeability in sedimentary rocks. Int J Rock Mech Min Sci 2009; 46(2): 229-238. 